\title{
Analyzing Unidentified Locked-Joint Failures in Kinematically Redundant Manipulators
}

\author{
Manish Goel* \\ School of Electrical and Computer Engineering \\ Purdue University \\ West Lafayette, Indiana 47907-1285 \\ Anthony A. Maciejewski ${ }^{\dagger}$ \\ Electrical and Computer Engineering Department \\ Colorado State University \\ Fort Collins, Colorado 80523-1373 \\ e-mail: aam@engr.colostate.edu \\ Venkataramanan Balakrishnan \\ School of Electrical and Computer Engineering \\ Purdue University \\ West Lafayette, Indiana 47907-1285 \\ Received 30 Juky 2003 ; accepted 2 Alogust 2004
}

\begin{abstract}
Robots are frequently used for operations in hostile environments. The very nature of these environments, however, increases the likelihood of robot failures. Common failurem tolerance techriques rely on effective failure detection and identification. Since a failure may not always be successfully identified, or, even if identified, may not be identified soon enough, it becomes important to consider the behavior of manipulators with unidentified failures. This work investigates the behavior of robots experiencing unidentified locked-joint failures in a general class of tasks characterized by point-to-point motion. Based on the analysis, a procedure for workspace evaluation is developed that allows for the identification of regions in the manipulator's workspace in which tasks may be completed even with such failures. (02005 Wiley Periodicals, Inc.
\end{abstract}

"presently at Applied Materials, Santa Clara, California 95050

tTo whom all correspondence should be addressed.

Joumal of Robotic Systems 22(1), 15-29 (2005) (c) 2005 Wiley Periodicals, Inc.

Published online in Wiley InterScience (wmw.interscience.wiley.com). - DOl: 10.1002/rob.20046 


\section{INTRODUCTION}

Failures in robots occur frequently in industrial operations. ${ }^{1}$ The likelihood of failures is far greater when robots are operated in harsh environments. ${ }^{2}$ Since the control of the individual joints is essentially independent in a typical robotic system, most failures affect only a single joint. While there are several ways in which a robot may fail, ${ }^{3-7}$ one common failure mode is a "locked joint," where the affected joint's velocity is identically zero. Such a failure may have catastrophic consequences or, at the very least, significantly degrade the system performance.

Since immediate human attention for repair or recovery is often not practical in hostile environments, it is desirable that the robot itself be able to cope with failures. A common approach to enhancing failure tolerance capability in a robot is the incorporation of redundancy in the design. This may be in the form of duplicated components ${ }^{8}$ or through the intelligent utilization of kinematic redundancy. ${ }^{9-13}$ Though there has been considerable work in the area of failure tolerant systems, there remain significant questions about the postfault behavior of robotic systems that do not incorporate fail-safe mechanisms. Existing failure tolerance schemes rely on effective failure detection and identification; ${ }^{14-16}$ only after a failure is identified is an appropriate failure recovery strategy initiated. ${ }^{17-21}$ However, failure identification is itself a difficult process that may not always be successful. ${ }^{3,22}$

While it is easy to envision a failure recovery strategy for identified failures, the situation is much more difficult when a failure is merely detected, but not identified. In particular, consider a joint malfunction where a significant deviation is detected between the commanded joint velocities and those returned by the joint sensors. Identifying whether this malfunction is due to a joint locking up or whether the sensor is (erroneously) returning a constant joint position is not possible. ${ }^{1}$ A simple response to such a situation is to implicitly assume that a joint has locked, and to adjust the control law accordingly so as to not command motion of the joint in question. ${ }^{23}$ However, given that sensor failures are more likely than joint actuation failures, ${ }^{22}$ this may not be the best course of action, as the workspace is unnecessarily reduced (often quite severeiy ${ }^{9,24,25}$ ).

We therefore consider the scenario where the control law remains unmodified, and explore the consequences. For the case when the sensor has indeed

${ }^{1}$ This assumes a typical commercial robot where duplicate sensing and/or analytical redundancy does not exist. failed, the ability of manipulators to converge to desired end-effector positions even with imperfect/ approximated Jacobians/Jacobian-inverses has been addressed in refs. 26 and 27 . Therefore, we focus on the other case where a joint has actually locked, but the controller continues to command motion of that joint as though it were healthy. For a general class of tasks characterized by point-to-point motion, we examine convergence issues such as whether the maripulator comes to rest and, if so, what is the terminal position and orientation of the end-effector. The convergence analysis is restricted to purely kinematic effects, due to the fact that the dynamic effects of a failure are essentially transient in nature and do not significantly affect the convergence behavior. $18,28-30$ Conditions under which the manipulator converges are explicitly defined and the anomalies in behavior due to the faults explained and illustrated with examples. These conditions are used to evaluate the convergence behavior of the manipulator over the postfailure workspace. This allows for the identification of workspace regions for which task completion may be possible even with unidentified failures.

\section{MATHEMATICAL FRAMEWORK}

The position and orientation ${ }^{2}$ of the end effector of a manipulator can be expressed in terms of its joint variables by the kinematic equation

$$
x=f(q),
$$

where $x \in \mathbb{R}^{m}$ is the position of the end effector, $q$ $\in \mathbb{R}^{n}$ is the vector of joint variables, and $m$ and $n$ the dimensions of the task space and joint space, respectively. Manipulators that have more degrees of freedom (DOFs) than required for a task, i.e., $n>m$, are said to be redundant. The end-effector velocity is expressed in terms of the joint rates as

$$
\dot{\mathrm{x}}=\int \dot{\mathrm{q}}
$$

where $J \in \mathbb{R}^{m \times n}$ is the manipulator Jacobian, $\dot{x}$ is the end-effector velocity, and $\dot{q}$ is the joint velocity.

If perfect servo control of the joints is assumed, then in a healthy manipulator the actual joint veloci-

"Elenceforth the term "position" will be used to mean any combi* nation of position and/or orientation variables. 
ties $\dot{\mathrm{q}}_{\mathrm{a}}$ equal the commanded velocities $\dot{\mathrm{q}}_{\mathrm{c}}$. However, in the event of a locked-joint failure of the ith joint, the corresponding element of $\dot{q}_{a}$ is identically zero. Then, the actual end-effector velocity is given by

$$
\dot{\mathrm{x}}_{\mathrm{a}}{ }^{\mathrm{i}} \mathrm{j} \dot{\mathrm{q}}_{\mathrm{c}}
$$

where ${ }^{i} J$ is the post-failure Jacobian, given by

$$
i^{i}=\left[\begin{array}{lllllll}
j_{1} & \cdots & j_{i-1} & 0 & j_{i+1} & \cdots & j_{n}
\end{array}\right]
$$

A common method for generating $\dot{\mathbf{q}}$ is the inverse kinematic scheme

$$
\dot{q}=G \dot{x},
$$

where $G$ is a generalized inverse of $J$ satisfying the Penrose condition $J G J=J$. A frequently encountered generalized inverse is the pseudoinverse $J^{+}$, which yields the least squares minimum norm solution. For full rank $J$, the pseudoinverse can be expressed as $y^{+}=J^{T}\left(U J^{T}\right)^{-1}$.

In this work, a general class of tasks characterized by sequences of point-to-point moves is considered. The commanded end-effector velocity is simply straight line motion towards the desired task position $x_{d}$ :

$$
\dot{x}_{c}=K_{e}\left(x_{d}-x_{a}\right)
$$

where $x_{a}$ is the actual position of the end effector, and $K_{e}$ is a constant position error gain that is adjusted when necessary to limit the commanded end-effector velocity to a maximum allowable value.

In the event of a locked-joint failure, the actual end-effector velocity in general will not be as commanded by (6). In particular, if joint $i$ fails, the actual end-effector velocity is given by

$$
\dot{x}_{\mathrm{a}}=\left({ }^{i} J G\right) K_{\mathrm{e}}\left(\mathbf{x}_{\mathrm{d}}-\mathbf{x}_{\mathrm{a}}\right)
$$

which can be obtained by combining (3), (5), and (6). Although $\dot{x}_{a}$ may not drive the end effector directly towards the desired task position, it is of interest to know whether the end effector will eventually converge to it. ${ }^{3}$

The first question that arises in the study of convergence behaviors of a failed manipulator is whether the desired task position is reachable, that is, lies within the postfailure workspace. If indeed it does, the issue of whether, under the employed control scheme, the manipulator converges correctly to the desired position must be examined. Even if reachability of the desired position is not possible for a certain failure, it may still be of interest to know whether the control allows the failed manipulator to converge to the position closest to that desired.

\section{POST-FAILURE REACHABILITY}

The workspace of a manipulator, in general, reduces after it experiences a failure., ${ }^{9,24}$ However, under certain conditions, a task position can be guaranteed to be reachable by a redundant manipulator after an arbitrary joint failure along a trajectory. This is achieved by ensuring that the ranges of the joint values along the nominal trajectory never exceed the bounds of the joint values given by the self-motion manifold(s) of the desired task position. ${ }^{24}$

The bounds of the self-motion manifolds can be directly computed by numerically tracing the manifold(s). Another more efficient way is to note that the extremal values of a joint variable are determined by the hyperplane that is tangential to the self-motion manifold with a normal that is collinear with the corresponding axis in the joint space. Consequently, the contribution of that joint to the null motion of the manipulator is identically zero. It is easily shown that a joint $i$ has a zero contribution to the null motion of the manipulator if and only if the Jacobian if $=\left[j_{1} \cdots j_{i-1} 0 j_{i+1} \cdots j_{n}\right]$ is rank deficient ${ }^{4}{ }^{12}$ If the condition for a joint to be at an extremum is not satisfied for any configuration corresponding to the desired end-effector position, then the range of that joint on the manifold is unconstrained, i.e., it spans the entire $\left[0^{\circ} 360^{\circ}\right]$ range. The limiting values of the range of joint $i$ are thus obtained by simultaneously solving the following equations:

$$
x_{d}=f(q)
$$

${ }^{3} \mathrm{~A}$ manipulator is considered to have converged when all its joints come to rest, t.e., all joints, except possibly the failed joint, are commanded a zero velocity by the employed control scheme.

${ }^{4}$ The rark deficiency of this matrix is referred to as a sentisingutarity in Ref. 31 . 
and

$$
\mathbf{n}_{I_{j}}(i)=0, \quad j=1, \ldots, n-m,
$$

where $\left\{\mathbf{n}_{J_{1}}, \ldots, \boldsymbol{n}_{J_{n-m}}\right\}$ represent any $n-m$ linearly independent null vectors of $J$.

The identified extrema partition the $\left[0^{\circ} 360^{\circ}\right]$ ranges of the joints into a number of segments. The segments corresponding to the joint ranges of the self-motion manifolds can then be identified by testing the midpoint of each of the obtained segments. Though this technique is a general one, its applicability depends upon the ease with which (8) and (9) can be solved.

The above techrique presents an efficient means for evaluating the effect of a given control scheme on the postfailure reachability of a task position. Moreover, the technique can also be employed for the design of control strategies to guarantee the reachability of critical task positions in a manipulator's workspace. ${ }^{24}$

\section{ANALYZING CONVERGENCE BEHAVIORS}

Independently of whether or not the reachability of a task position is guaranteed for a failure, it is important to analyze the convergence behavior of a failed manipulator for a given control scheme. If the desired task position is reachable, will the failed manipulator converge to that position? If it is not reachable, will the failed manipulator converge to the closest possible position? If it does not converge to one of these positions, to what position does it converge, if it does converge at all? These issues form the crux of the analysis of the postfault behavior of manipulators.

We will define "convergence" formally to mean that the joints come to rest, that is, $\dot{\mathrm{q}}_{\mathrm{a}}=0$. This is possible for a failed manipulator if either all its joints are commanded zero velocities, or if only the failed joint(s) are commanded motion (since $\dot{\mathrm{q}}_{a}={ }^{i} \dot{\mathrm{q}}_{\mathrm{c}}$ ). In the context of the defined control framework, convergence with zero velocities of all the manipulator joints is possible only if the commanded end-effector velocity is zero. Since a zero end-effector velocity is commanded only if the end effector is at the desired position, convergence with $\dot{\mathrm{q}}_{\mathrm{c}}=0$ implies correct convergence. Convergence with $\dot{\mathrm{q}}_{\mathrm{c}} \neq 0$ (i.e., when some or all of the failed joints are commanded nonzero velocities), therefore, must imply "erroneousconvergence" or convergence to a position other than the desired one. To determine the factors character- izing erroneous convergence of a manipulator, it is necessary to identify conditions under which only the failed joints of the manipulator are commanded motion.

\subsection{Characterizing Convergence}

The following theorem shows that a manipulator gets "stuck," or converges with a non-zero commanded velocity, onty when the following two conditions hold:

- The commanded end-effector velocity lies in the space spanned by the failed columns of the Jacobian.

- The post-failure weighted Jacobian is rank deficient.

This result is derived as follows:

Theorem 1: Consider a manipulator at a nonsingular configuration, driven by a generalized inverse control

$$
\dot{\mathrm{q}}_{\mathrm{c}}=G \dot{\mathrm{x}}_{\mathrm{c}}
$$

where $G=W^{-1} J^{T}\left(J W^{-1} J^{T}\right)^{-1}$ for some symmetric $W>0 .{ }^{5}$ Let $S$ be the set of the indices of the $k$ locked joints, and let $j_{i}$ and $w_{i}$ denote the $i$ th columns of $J$ and $W$, respectively. Then,

1. Only the failed joints are commanded motion if and only if the commanded end-effector velocity vector $\dot{x}_{c}$ lies in the space spanned by the columns corresponding to the failed joints of the Jacobian, i.e.,

$$
\dot{\mathrm{q}}_{\mathrm{c}}=\sum_{i \in S} \alpha_{i} \mathrm{e}_{i} \Leftrightarrow \dot{\mathrm{x}}_{\mathrm{c}}=\sum_{i \in S} \alpha_{i} \dot{j}_{i}
$$

for some $\alpha_{i} \in \mathbb{R}, i \in S$, where $\mathbf{e}_{i}$ represents a natural basis vector.

2. Moreover, the failed joints are commanded nonzero velocities only if a postfailure weighted Jacobian is rank deficient, i.e.,

$$
\dot{\mathrm{q}}_{\mathrm{c}}=\sum_{i \in S} \alpha_{i} \mathrm{e}_{i} \neq 0 \Rightarrow{ }^{i}\left(J W^{-1}\right) \text { rank deficient }
$$

${ }^{5}$ The matrix $W$ is used to specify the relative weighting on the joint velocities when determining the minimum norm solution. 
where ${ }^{i}\left(J W^{-1}\right) \in \|_{2}^{m \times(n-k)}$ is obtained from $J W^{-1}$ by zeroing the columns with indices $i \in S$.

Proof:

1. The condition

$$
\dot{\mathrm{q}}_{\mathrm{c}}=\sum_{i \in S} \alpha_{i} \mathbf{e}_{i} \Rightarrow \dot{\mathrm{x}}_{c}=\sum_{i \in S} \alpha_{i} \dot{j}_{i}
$$

can be established simply by premultiplying the equation on the left by $J$.

For the converse the following is given:

$$
\dot{x}_{c}=\sum_{i \in S} \alpha_{i} j_{i}
$$

Let $N \in \mathbb{R}_{(n-m) \times n}$ be a matrix whose columuns span the null space of $J$. Then, for the given inverse control

$$
N^{T} W \dot{q}_{c}=0
$$

Using the fact that $\dot{x}_{c}=J \dot{q}_{c}$, the two equations above can be written in a matrix form as

$$
\left[N^{T} W\right] \dot{q}_{c}=\left[\begin{array}{c}
\Sigma_{i \in S} \alpha_{i} \dot{j}_{i} \\
0
\end{array}\right] .
$$

The matrix on the left is full-rark and invertible and so the system of equations has a unique solution. Since $\dot{\mathrm{q}}_{\mathrm{c}}=\Sigma_{i \in S} \alpha_{i} \mathrm{e}_{i}$ is a solution, it must be the unique solution. This completes the argument.

2. The second statement of the theorem is proved by contradiction. Suppose that for some nonzero $\dot{q}_{c}$, ' $\left(J W^{-1}\right)$ is full-rank. From the first statement of the theorem, $\dot{\mathbf{q}}_{\mathrm{c}}=\Sigma_{i \in S} \alpha_{i} \mathrm{e}_{i}$. Then, from $N^{T} W \dot{q}_{\mathrm{c}}=0$, it follows that $N^{T}\left(\Sigma_{i \in S} \alpha_{i} \mathbf{w}_{i}\right)=0$. This implies that $\Sigma_{i \epsilon S} \alpha_{i} w_{i} \in \mathcal{R}\left(J^{T}\right)$ or, equivalently, that

$$
W \sum_{i \in S} \alpha_{i} \mathrm{e}_{i}=\mathrm{J}^{\mathrm{T}} \mathbf{v}
$$

for some $\mathrm{v} \neq 0$. This can be rewritten as

$$
\sum_{i \Subset S} \alpha_{i} \mathrm{e}_{i}=\left(J W^{-1}\right)^{T} \mathbf{v}
$$

Zeroing out the rows with indices $i \in S$ (corresponding to the failed joints) on both sides of this equation yields

$$
0={ }^{i}\left(J W^{-1}\right)^{T} v
$$

This implies that ${ }^{i}\left(J W^{-1}\right)^{T}$ has a nontrivial null space and therefore is rank deficient. Thus ${ }^{i}\left(J W^{-1}\right)$ is also rank deficient-a contradiction.

The second statement of the theorem says that if the rank-deficiency condition does not hold at a configuration, then the manipulator cannot come to rest at that configuration with a nonzero commanded joint velocity.

Theorem 1 identifies the various parameters that affect the convergence of the manipulator after unidentified joint failures: the joints that have failed, the configurations at which they failed, the actual position of the end effector, the desired task position for the end effector and the inverse-kinematic control employed. It is important to point out that Theorem 1 only characterizes potential positions of convergence, and does not answer the question of whether the manipulator will eventually converge to such a configuration and, if so, to which one. However, conditions under which each of the following three distinct behaviors may be exhibited can be inferred from the theorem:

1. The manipulator successfully converges to the desired task position.

2. The manipulator converges, but to a position other than the desired task position.

3. The manipulator does not converge, i.e., it keeps moving forever.

Each of these behaviors can be encountered under normal operation, as will be demonstrated in Sections 4.2 and 4.3 .

\subsection{A 2-DOF Planar Example}

Since the workspace of a planar 2-DOF manipulator reduces in dimension after either joint failure, the postfailure reachability of a task position is almost always not possible. This simple example, however, is ideal for a detailed analysis of the postfailure convergence behavior where, in addition, a "brute-force" analysis of convergence can be performed. All three 


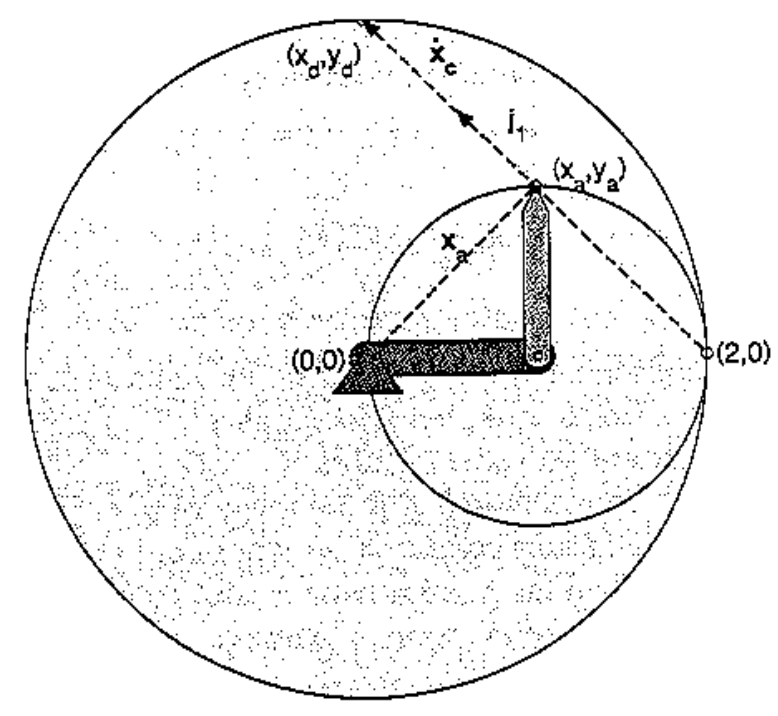

Figure 1. Geometric interpretation of the convergence condition from Theorem 1 for the 2-DOF example. Since $\left.{ }^{1}\right]$ is always rank deficient for the 2-DOF manipulator, the maripulator converges when $\dot{x}_{c}$ and $j_{1}$ are collinear. Thus, motion of only joint 1 (failed) is commanded, and, therefore, the manipulator does not move.

behaviors listed in Section 4.1 are illustrated here for the case of a simple unit link-length, planar 2-DOF manipulator. The failure of the first joint at $0^{\circ}$ is considered (without loss of generality).

For joint variables $\theta_{1}$ and $\theta_{2}$, the velocity relation between the end effector and the joint variables given by (2) can be written as

$$
\dot{\mathrm{x}}_{\mathrm{a}}=J\left[\begin{array}{c}
\dot{\theta}_{1} \\
\dot{\theta}_{2}
\end{array}\right]
$$

where the Jacobian Y expressed in the base coordinate frame is

$$
J=\left[\begin{array}{cc}
-s_{1}-s_{12} & -s_{12} \\
c_{1}+c_{12} & c_{12}
\end{array}\right]
$$

with $s_{1}=\sin \left(\theta_{1}\right), c_{1}=\cos \left(\theta_{1}\right), s_{12}=\sin \left(\theta_{1}+\theta_{2}\right)$, and $c_{12}$ $=\cos \left(\theta_{1}+\theta_{2}\right)$.

Let the task position be given by $x_{d}=\left[x_{\mathrm{d}} y_{\mathrm{d}}\right]^{T}$, where $x_{d}$ is not restricted to be either in the postfailure or the original workspace. When the manipulator

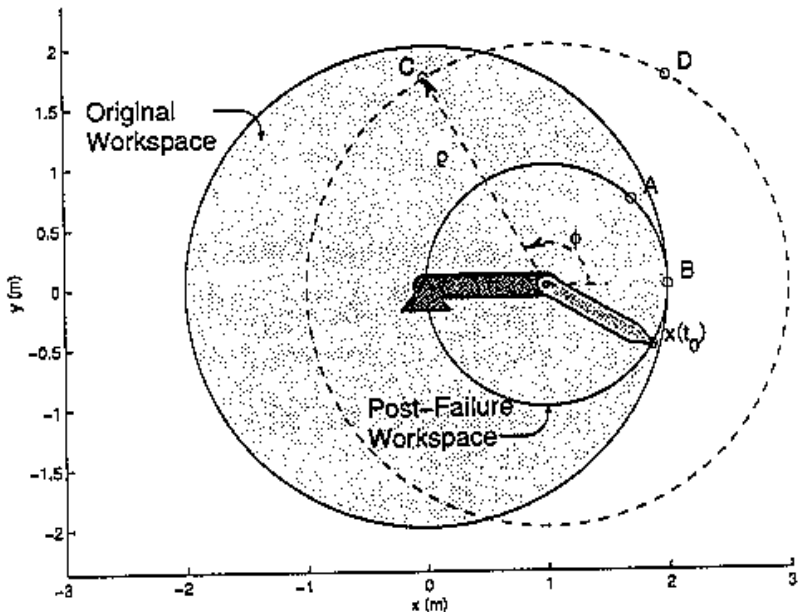

Figure 2. A 2-DOF planar manipulator with joint 1 failed at $0^{\circ}$. The shaded area represents the workspace of the healthy manipulator that reduces to the inner circle after the failure. Also shown in this plot are four task positions A, B, C, and D for which very different convergence behaviors are observed for the same starting position $x\left(t_{0}\right)$.

is driven by the control input ${ }^{6}$ defined in (6), with a unit value of the positional error gain $K_{e}$, the velocity of joint 2 , after a locked-joint failure of joint 1 , is given by

$$
\dot{\theta}_{2}=\frac{1}{\sin \theta_{2}}\left(\left(2-x_{\mathrm{d}}\right)\left(1+\cos \theta_{2}\right)-y_{\mathrm{d}} \sin \theta_{2}\right)
$$

The condition for convergence is $\dot{\theta}_{2}=0$, which results, from simple trigonometry, in

$$
\frac{x_{\mathrm{a}}}{y_{\mathrm{a}}}=-\frac{y_{\mathrm{d}}}{x_{\mathrm{d}}-2}
$$

This condition can be reinterpreted in light of Theorem 1. From Figure 1, it is seen that condition (16) is equivalent to the position vector $x_{a}$ and the commanded end-effector velocity $\dot{x}_{c}$ being orthogonal. Since $j_{1}$ and $x_{a}$ are also orthogonal, it can be concluded that $\dot{x}_{c}$ and $j_{t}$ must be collinear. Thus, the motion of only joint $I$ (failed) is commanded. This is precisely the convergence condition postulated by Theorem 1. It should be noted that for the 2-DOF ma-

${ }^{6}$ Here $G$ is simply the inverse of the Jacobian, which is uniquely defined except at the singularities of the manipulator identified by the configurations where $\theta_{2}=k \pi, k$ integer. 


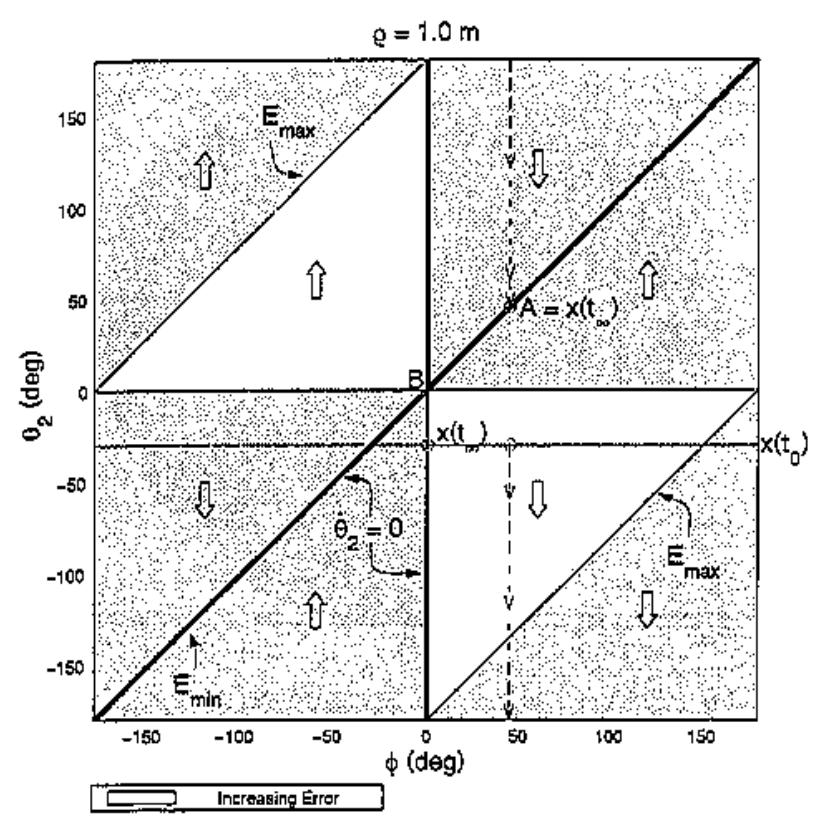

Figure 3. Joint-space trajectory evolutions for task positions that are located $1 \mathrm{~m}$ from the $\theta_{2}$ joint axis, e.g., task positions $\mathrm{A}$ and $\mathrm{B}$. The large arrows denote the evolution of the trajectory in a region. The end effector position error increases in the light-gray regions, and decreases in the dark-gray regions. The dark lines indicate configurations to which the manipulator may converge, i.e., the commarded joint two velocity will be zero. Convergence to the task position is observed. with $A$. No motion results when the task position is $\mathbf{B}$.

ripulator the rank-deficiency condition of the theorem always holds since ${ }^{1} J$ and ${ }^{2} J$ are always rank deficient. Thus, for this example, only the collinearity condition determines convergence.

It is seen that Theorem 1 only characterizes potential positions of convergence, and does not answer the questions of whether the manipulator will eventually converge to such a configuration, and, if so, to which one. To address this issue, the evolution of the manipulator trajectory is investigated next for different task positions. For this analysis, it is convenient to represent $x_{\mathrm{d}}$ and $y_{\mathrm{d}}$ in polar-coordinates $(\rho, \phi)$ with origin at joint 2 :

$$
x_{\mathrm{d}}=\rho \cos (\phi)+1, \text { and } y_{\mathrm{d}}=\rho \sin (\phi) \text {. }
$$

Four task positions that exhibit very different convergence behaviors are $\mathrm{A}=\left(1,45^{\circ}\right), \mathrm{B}=\left(1,0^{\circ}\right)$, $\mathrm{C}=\left(2,120^{\circ}\right.$ ), and $\mathrm{D}=\left(2,60^{\circ}\right)$ (see Figure 2$)$. In each case, the end effector is initially positioned at $\mathbf{x}\left(t_{0}\right)=(1$,

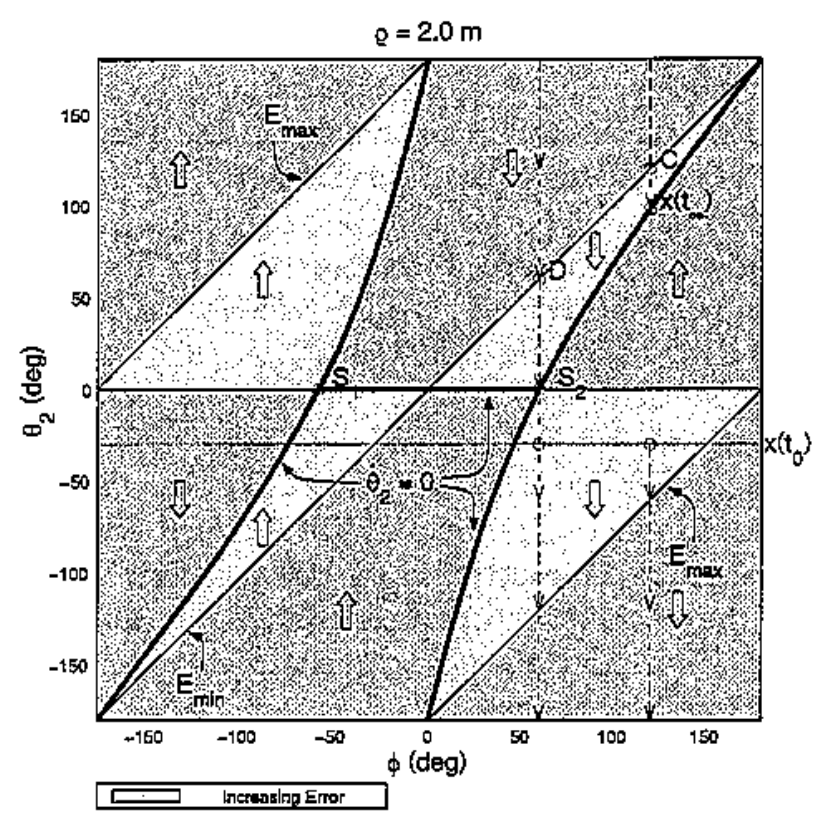

Figure 4. Joint-space trajectory evolutions for task positions that are located $2 \mathrm{~m}$ from the $\theta_{2}$ joint axis, e.g., task positions $C$ and $D$. The large arrows denote the evolution of the trajectory in a region. The end effector position error increases in the light-gray regions, and decreases in the dark-gray regions. The dark H-shaped curve indicates configurations to which the manipulator may converge, i.e., the commanded joint two velocity will be zero. Note that the line corresponding to configurations that are closest to the desired task positions (denoted $E_{\text {min }}$ ) does not coincide with the possible configurations of convergence. Thus, the end effector converges to a position that is not closest to C. For task position D, the manipulator does not converge at all, but cycles continuously.

$-30^{\circ}$ ), with the robot configuration given by $\theta_{1}$ $=0^{\circ}$ and $\theta_{2}=-30^{\circ}$, and is commanded to move toward the task position until convergence. The jointspace trajectories for these cases are plotted in Figures 3 and 4 as functions of $\theta_{2}$ and $\phi$ (since $\theta_{1}$ is always 0 ) for $\rho=1$ and $\rho=2$, respectively. For each particular task position, $\phi$ is constant and the trajectory evolves along the $\theta_{2}$ axis. For a given $\phi$, the task positions are characterized in these plots by $\theta_{2}=\phi$. Points of convergence are at the intersection of the trajectory of $\theta_{2}$ with the task position, or with the $\dot{\theta}_{2}=0$ curve. $^{7}$

Both the end-effector position error $E=\left\|x_{\mathrm{d}}-\mathrm{x}_{\mathrm{a}}\right\|$ and its rate of change $\dot{E}$ are shown in the plot. The minimum and the maximum contours of the error $E$

In Figure 4, the $\dot{\theta}_{2}=0$ curve is discontinuous at positions $S_{1}$ and $S_{2}$. For the given value of $\rho=2.0, \dot{\theta}_{2}$ is nonzero at these positions [from (15)]. 
are denoted by $E_{\min }$ and $E_{\max }$, respectively. In the dark-gray regions, $\dot{E}$ is negative, and it is positive in the light-gray regions. In each region, the evolution of $\theta_{2}$ is governed by (15) and is indicated in the plots by large arrows.

Case 1. Successful convergence. From Figure 3, it can be seen that for almost all $\phi_{\text {, the }} \theta_{2}$ trajectory is drawn to the task position $\theta_{2}=\phi$. [One such example is shown with point $\mathrm{A}\left(\phi=45^{\circ}\right)$.] This behavior is exhibited only for $p=1$, i.e., when the task position lies in the postfailure workspace. It will be seen that for any other value of $\rho$, not only will successful convergence not be achieved (since the task position lies outside the postfailure workspace), but also the manipulator will almost always not converge to the point closest to that desired.

Even though successful convergence occurs for $\rho=1$, the path taken by the end effector is clearly not always the shortest. Also, the end-effector error initially increases for large regions of $\theta_{2}$ and $\phi$, leading to large excursions of joint 2 , which may violate joint limit constraints and therefore prevent convergence. It is also important to note that such behavior is not preventable by simply restricting only the workspace (by limiting $\phi$ ) or only the robot configuration (by constraining $\theta_{2}$ ).

Case 2. Erroneous convergence. Two types of erroneous convergence are seen. The first is the unique situation where the task position (B) is in the postfailure workspace, but the robot does not move at all (see Figure 3). Here, $\dot{x}_{c}$ and $j_{1}$ are collinear and $\dot{\theta}_{2}$ $=0$. Indeed this condition is true for any $\theta_{2}$, and therefore the robot does not move for any initial configuration. Note that this behavior will be observed at every point on the boundary of the original workspace if joint $I$ fails with the appropriate value for $\theta_{1}$.

The second, more common, type of erroneous convergence is observed when the task position is outside the postfailure workspace. Here, the end effector does move, but converges to a point other than one that minimizes the error, i.e., $\theta_{2}=\phi$. One such example is the point $\mathrm{C}$ shown in Figure 4 . The figure indicates that even for a constant value of $\rho$, very different errors may be observed at convergence depending upon the value of $\phi$. The error is a minimum for $\phi=180^{\circ}$, and rises to a maximum for $\phi$ close to $60^{\circ}$. For the special case when $\phi=60^{\circ}$, no convergence occurs; this is discussed next.

Case 3. No convergence. $D$ is an example of a task position that lies outside the original workspace,

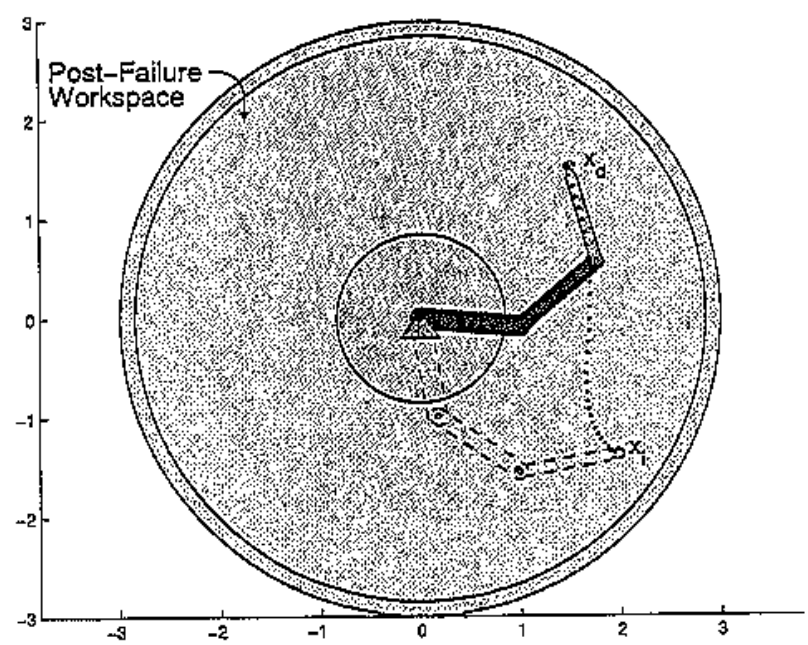

Figure 5. Successful convergence to the task position. The maripulator is able to complete the point-to-point motion task between $x_{i}$ and $x_{d}$, even with a failure of joint 2 ( $x_{i}$ $=[2.0-1.4]^{\mathrm{T}}$ and $x_{\mathrm{d}}=[1.51 .5]^{\mathrm{T}}$ ).

and on the line $x_{\mathrm{d}}=2$. For such points, (15) gives $\dot{\theta}_{2}$ $=-y_{d}$. Thus, the manipulator rotates with a constant angular velocity.

Remarks: Joint-space plots such as those in Figures 3 and 4 are valuable workspace layout tools. As will be illustrated in Section 4.5 , these plots can be used to identify regions of convergence where successful task completion can be achieved even in the presence of unidentified joint failures.

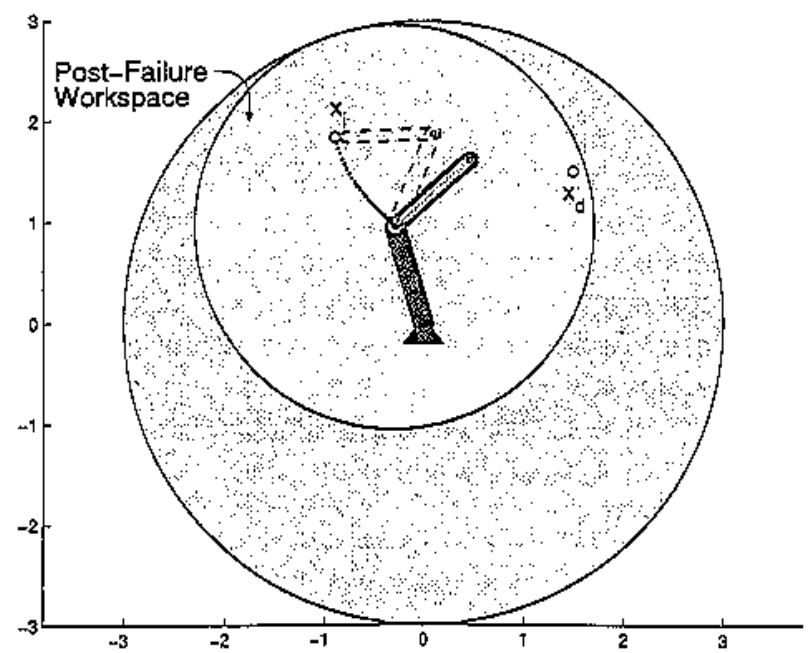

Figure 6. Erroneous convergence when the desired task position $x_{d}$ is in the postfailure workspace. With a joint 1 failure the manipulator converges to a configuration where the "reduced-manipulator" becomes singular ( $1 \mathrm{~J}$ $=\left[j_{2} j_{3}\right]$ rank deficient), and $\dot{x}_{c}$ and $j_{1}$ align ( $x_{i}$ $=[-0.91 .8]^{T}$ and $x_{d}=[1.51 .5]^{T}$ ). 


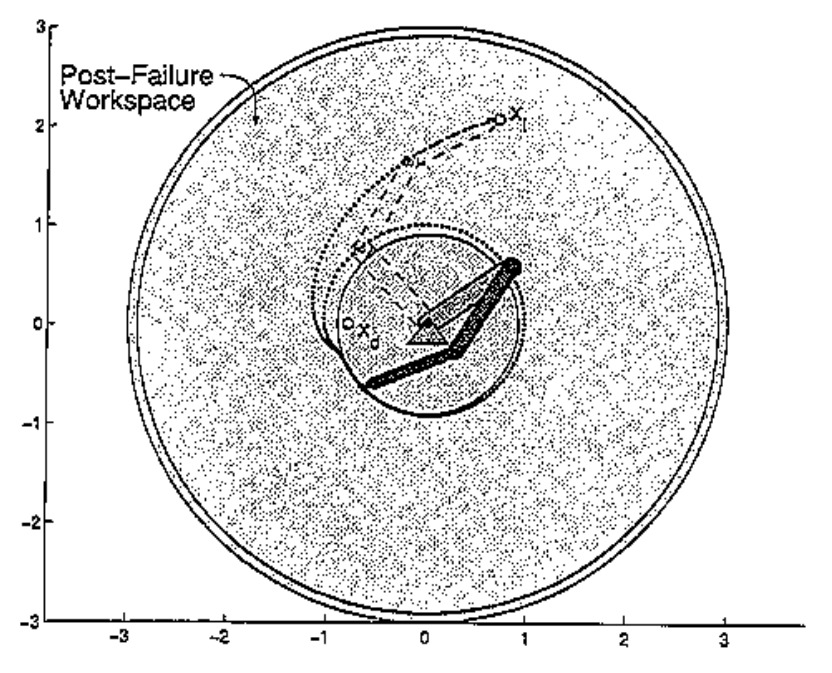

Figure 7. No convergence. With the choice of inversekinematics used, nonzero joint velocities are commanded in the healthy joints everywhere, therefore the manipulator does not converge with the Joint 3 failure $\left(x_{i}\right.$ $=[0.72 .1]^{T}$ and $x_{d}=[-0.80]^{T}$ ).

\subsection{A 3-DOF Planar Example}

Kinematic redundancy can be used to guarantee the postfailure reachability of task positions. However, potential problems with postfailure convergence remain even in this case, as will be demonstrated with a planar 3-DOF example. All three distinct convergence behaviors are illustrated here with three different cases shown in Figures 5-7. The failures of different joints are considered in these examples, and pseudoinverse control is assumed. In each of these cases the failed manipulator performs a point-topoint motion task from an initial position $x_{i}$ to a desired task position $x_{d}$. In the first case, shown in Figure 5 , the manipulator is able to successfully complete the task even with a failure of joint 2. Illustrated in Figure 6 is a case of erroneous convergence, where, even though the desired task position $x_{d}$ is the same as in Figure 5 (and lies in the post-failure workspace after a failure of joint 1), the manipulator converges elsewhere. Presented in Figure 7 is a case where no convergence is observed for the failure of joint 3 . In this case, the desired task position lies in the original workspace, but outside the postfailure workspace. However, since at no position in the postfailure workspace are the conditions necessary for convergence satisfied, convergence is not possible. The manipulator therefore moves continuously.

The different convergence behaviors identified and illustrated above are characterized by the properties of the configurations to which a manipulator erroneously converges. An analysis of these configurations is thus necessary for a better understanding of the postfault behavior of the manipulator.

\subsection{Conditions for Erroneous Convergence}

The conditions of Theorem 1 can be used to determine configurations of erroneous convergence for a failed manipulator. Suppose the column of the Jacobian corresponding to a failed revolute joint $i$ is expressed in standard Denavit-Hartenberg notation as

$$
\mathbf{j}_{i}=\left[\begin{array}{c}
\hat{\mathbf{z}}_{i-1} \times \mathbf{p}_{i} \\
\hat{\mathbf{z}}_{i-1}
\end{array}\right]
$$

where coordinate frame $(i-1)$ is attached to link ( $i$ -1 ), the motion of joint $i$ is along the $\hat{z}_{i-1}$ axis, and $p_{i}$ represents the position vector of the end effector expressed in the coordinate frame $(i-1)$. Then, if $\dot{\mathbf{x}}_{v}$ and $\dot{x}_{\omega}$ represent the translational and rotational components of $\dot{x}_{c}$, the first condition is equivalent to simultaneously satisfying the following conditions:

$$
\dot{\mathbf{x}}_{v}^{T} \mathbf{p}_{i}=\dot{\mathbf{x}}_{v}^{T} \hat{\mathbf{z}}_{i-1}=0
$$

and

$$
\dot{\mathrm{x}}_{\omega}^{\mathrm{T}} \hat{\mathbf{x}}_{i-1}=\dot{\mathrm{x}}_{\omega}^{\mathrm{T}} \hat{\mathrm{y}}_{i-1}=0
$$

With pseudoinverse control $(W=I)$, the rank deficiency condition in Theorem 1 is equivalent to the $i$ th component of each of the $n-m$ linearly independent null vectors of $J$ being identically zero. ${ }^{12}$ Thus if $\left\{\mathrm{n}_{f_{1}}, \ldots, \mathrm{n}_{I_{n-m}}\right\}$ represent any $n-m$ linearly independent null vectors of $J$, then the second condition of Theorem 1 is equivalent to

$$
\mathrm{n}_{f_{j}}(i)=0, \quad j=1, \ldots, n-m
$$

Equations (19)-(21) represent the conditions that must hold for erroneous convergence. For a given failure angle of a joint, these equations can be solved for the other joint variables to determine the problematic configurations. 


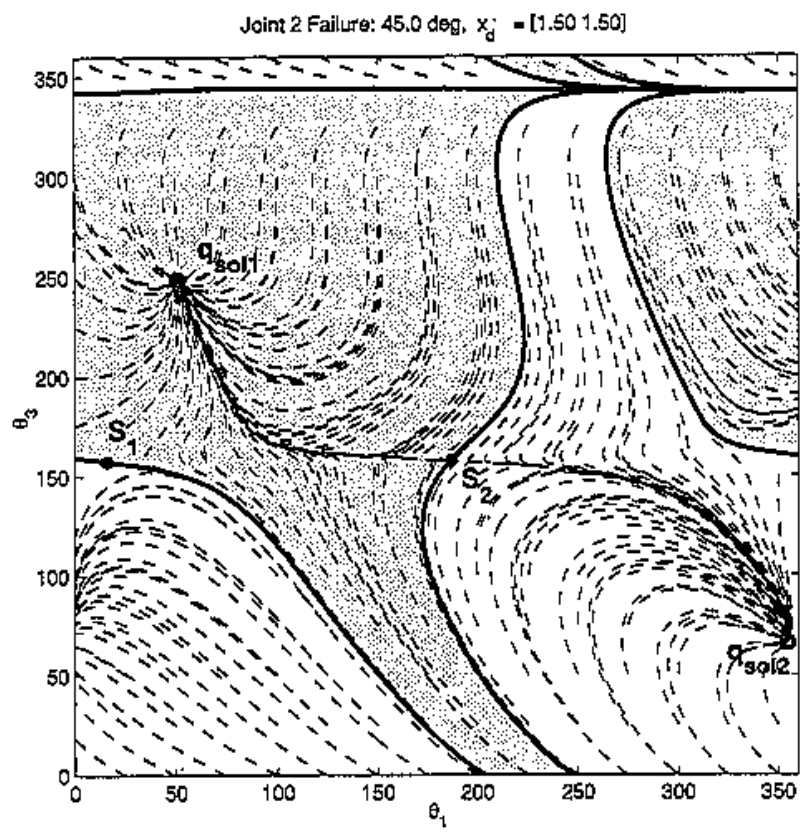

Figure 8. Joint-space trajectories of a 3-DOF unit link length, planar manipulator, with a failure of Joint 2 at $45^{\circ}$. $S_{1}$ and $S_{2}$ represent the two stationary configurations, while $\mathrm{q}_{\mathrm{sol}}$ and $\mathrm{q}_{\mathrm{sol}}$ represent the two post-failure solutions corresponding to the desired position. The solid lines represent trajectories that are drawn to the stationary points; they also partition the joint space into the two basins. All trajectories (shown by dashed lines) that originate in the gray basin converge to $\mathrm{q}_{\text {sol }_{1}}$, while those that originate in the unshaded basin converge to $\mathrm{q}_{\mathrm{sol}_{2}}$.

\subsection{Analyzing Stationary Configurations}

After the stationary configurations of a manipulator are determined, an important question is whether a manipulator can be drawn into any of these configurations after a failure. The local behavior of the failed manipulator about a stationary point $\left(\mathrm{q}_{0}\right)$ can be determined by analyzing the stability of the linearized system dynamics about the point. For $\dot{\mathrm{q}}=g(\mathrm{q})$, where $g(q)={ }^{i} I G K_{c}\left(x_{d}-x_{a}\right)$, linearization about $q_{0}$ yields

$$
\frac{d}{d t} \Delta \mathrm{q}=\left(\left.\frac{\partial g}{\partial \mathbf{q}}\right|_{q_{0}}\right) \Delta \mathrm{q}=\tilde{J} \Delta \mathrm{q} .
$$

Since the components of the vectors $\Delta \mathrm{q}$ and (d/dt) $\Delta \mathrm{q}$ corresponding to the failed joint $i$ are identically zero at all times after the failure, the matrix $\tilde{j}_{i}$, obtained from $\bar{y}$ by removing the components corresponding to joint $i$, is examined for stability. While an
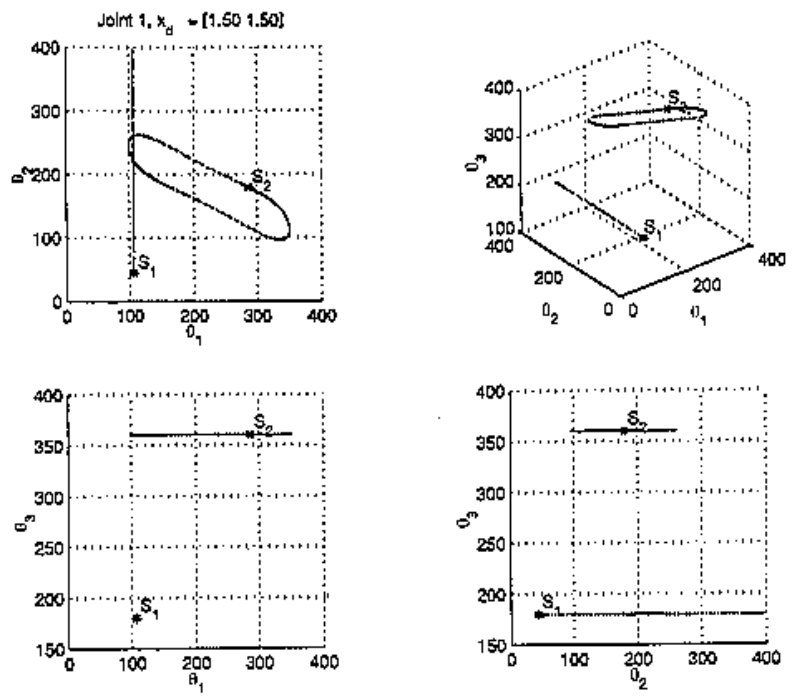

(a)
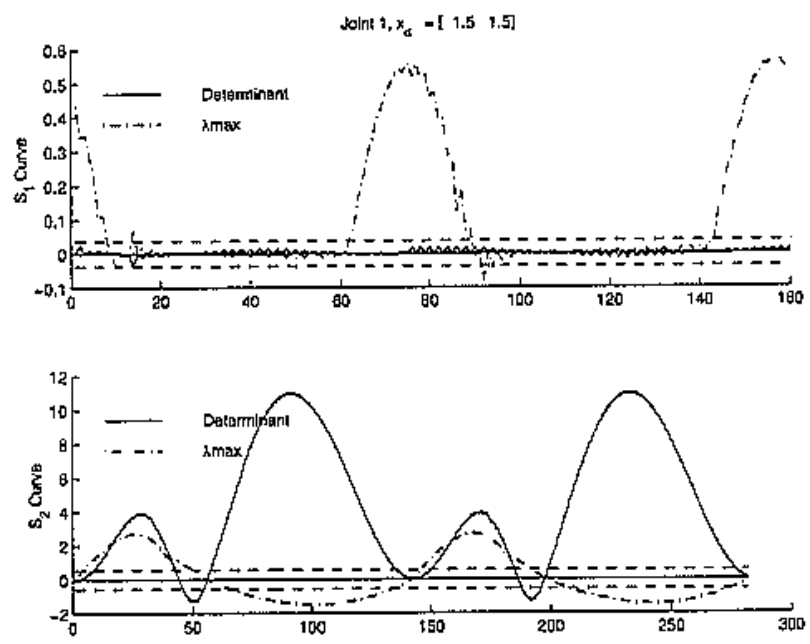

(b)

Figure 9. Stationary curves for joint 1 failures of a 3-DOF unit link length planar manipulator are shown in (a). $S_{1}$ and $S_{2}$ denote the two seed configurations used to trace these curves. The sink segments are shown using dotted lines, while the nonsink segments are shown with solid lines. The segments are partitioned based on the behavior of the determinant and the real part of the eigenvalues of $\tilde{J}_{1}$ shown in (b). The dashed lines in (b) defire a "zero band" that is used to check for the zero crossings of the determinant curve.

antistable equitibrium point (where all eigenvalues of $\bar{J}_{i}$ have positive real parts) poses no problem as far as erroneous convergence of the manipulator is concerned, a stable equilibrium point (all eigenvalues have negative real parts) acts as an attractor and is a potential problem. A stable stationary configuration 


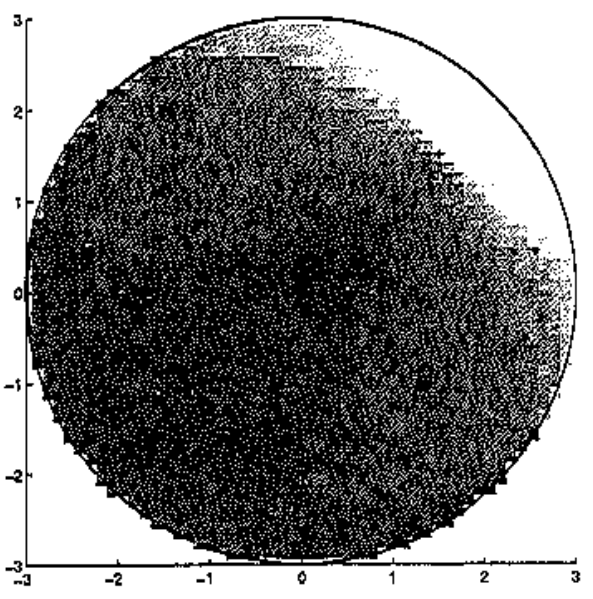

(a) $\mathcal{R}_{100}$

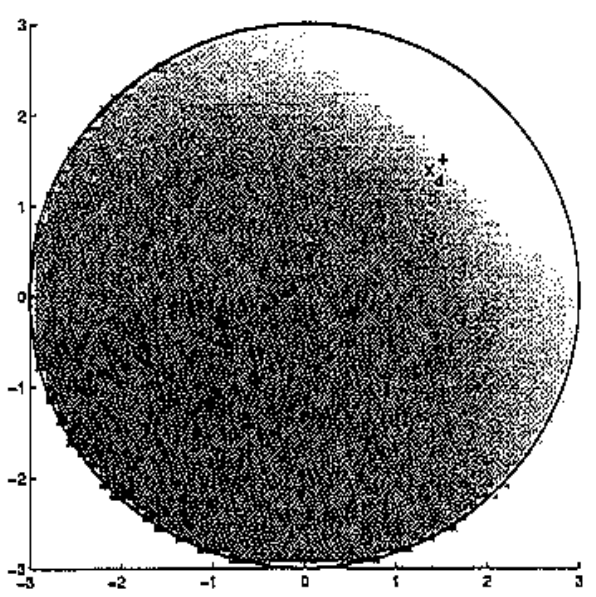

(c) $\mathcal{R}_{\mathrm{avg}}$

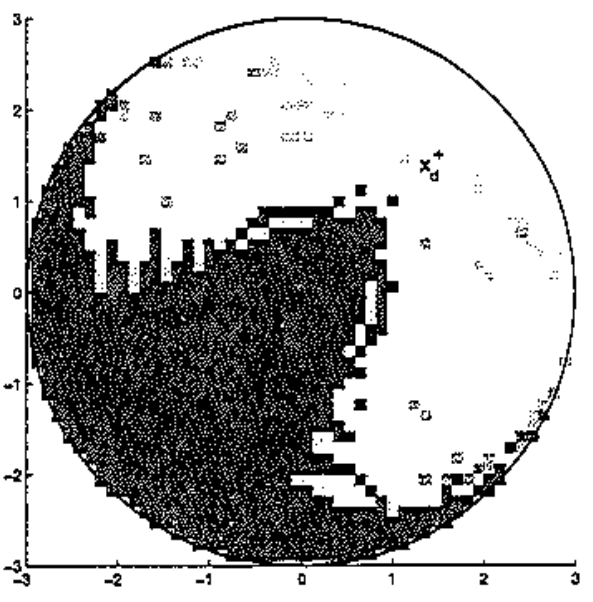

(b) $\mathcal{F}_{100}$

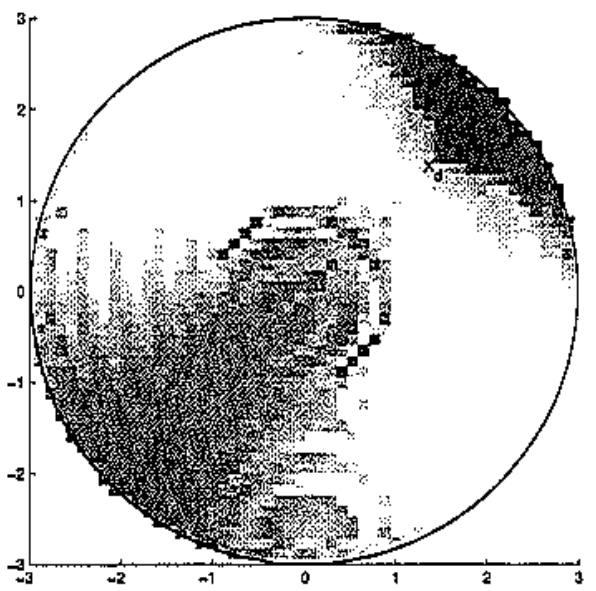

(d) $R_{\text {worss }}$

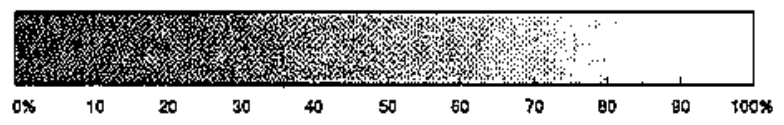

Figure 10. Workspace shaded as a function of, $\mathcal{R}_{100}, \mathcal{F}_{100}, \mathcal{R}_{\mathrm{jvg}}$, and $\mathcal{R}_{\mathrm{sens}}$, for arbitrary joint failures $\left(x_{\mathrm{d}}=[1.51 .5]^{\mathrm{T}}\right)$.

is classified as a "sink." For an unstable equilibrium point (some eigenvalues have negative real parts), the stable modes can be used to trace the failure configurations for which the manipulator gets drawn to a stationary point.

These traces, which are in general manifolds, may in some cases partition the workspace into distinct regions or "basins," as seen for the 3-DOF example in Figure 8. Since trajectories originating off the basin boundaries can never reach these botundaries, all trajectories are confined to the basins where they originate. Note that the Cartesian-space trajec- tory shown in Figure 5 is an example corresponding to a configuration that lies in the unshaded basin; it thus converges to solution $\mathrm{q}_{\mathrm{si}_{2}}$.

It is important to point out that urless the stationary points are sinks, the dimension of the manifolds traced along the stable mode(s) is at least two less than the dimension of the joint space (or the number of DOFs) of the manipulator. This is because the failure itself constrains one variable, reducing the dimension by one, while the additional reductions in dimension, if any, result due to the unstable modes. This implies that in the absence of sinks, the probabil- 
ity of a failure resulting in a maripulator configuration on a problematic manifold is zero.

\subsection{Tracing and Classifying Stationary Manifoids}

For an analysis of correct convergence, all potentially problematic stationary configurations in the manipulator's joint space must be identified. The loci of these stationary configurations (referred to as "stationary manifoids") must be generated for every possible joint failure. The first step in tracing a stationary manifold for a particular joint is to identify one stationary configuration for each distinct singularity of the failed arm. These stationary configurations represent the set of seed configurations that are grown to trace out the entire stationary manifold. Since (19)(21) must hold at all stationary configurations, the loci of stationary configurations can be traced by simply starting at a seed that satisfies these conditions, and moving in a direction orthogonal to the gradients of the right-hand side components of these equations. Doing this for each seed allows all the loci to be traced. More specifically, a matrix $M$ is formed by collecting the gradients, and joint motion in the null space of $M$ traces the manifolds [see Figure 9(a)]. The rank of $M$ may drop along a trace, signifying the increase in the dimension of the null space, and hence a branching in the manifold. This is an issue that must be addressed in the implementation of this techrique.

Sinks, being the real hindrance to correct convergence, must be identified on the stationary manifolds. This is done by checking the eigenvalues of $\tilde{J}_{i}$ at each point on the discretized manifolds. For manipulators with exactly one degree of redundancy (as in the 3-DOF example considered), $\tilde{J}_{i}$ is square, and in such cases the partitioning process can be performed more efficiently by evaluating the determinant of $\tilde{J}_{i}$, and checking the eigenvalues only at the zero crossings of the determinant. This is because orly at these zero crossings can the eigenvalues change sign. The stationary manifolds can thus be partitioned into "sink" and "nonsink" segments [see Figure 9(b)].

\section{WORKSPACE ANALYSIS}

The postfailure convergence behavior of manipulators, in addition to being functions of other variables,

${ }^{8}$ Tracing stationary manifolds in high dimensions is in general a difficult problem. While there exist techniques for tracing $2-D$ manifolds, such as the one presented in ref. 24, the formulation of general methods to trace such surfaces in ligher dimensions is an open problem. is seen to be dependent on task placement. If failure identification is not available, or otherwise, not reliable, this knowledge can be exploited to better cope with failures. Through the characterization and classification of workspace regions, critical tasks can be placed in locations to which the manipulator can correctly converge even after an arbitrary joint failure.

Analyzing the workspace of a manipulator for failure toletance involves checking for reachability and correct convergence. Based on the ideas presented in the previous sections, a systematic procedure can be developed to analyze a manipulator's workspace. For the given task model of point-topoint motion, a specified control scheme, and a desired task position, it is of interest to evaluate the entire workspace to determine the effect of the initial task position on the convergence behavior of a maniptulator when anticipating unidentified locked-joint failures. Some concerns when evaluating a candidate initial task position are the following.

- For what percentage of the trajectories originating on the self-motion manifolds of the initial position can reachability of the desired position be guaranteed? If reachability cannot be guaranteed for a failure along the entire trajectory, then for what percentage of the trajectory can reachability be guaranteed?

- How much does the percentage reachability change with the initial configuration? That is, how sensitive is reachability to a choice of the initial configuration?

Similarly, questions about correct convergence to the desired position can be posed for trajectory portions guaranteeing reachability.

For a given desired task position, the following procedure allows the workspace to be evaluated for identifying the suitability of different initial positions for the task. ${ }^{9}$ The first step of the procedure is to discretize the entire workspace. The discretized positions in the workspace represent the set of initial positions to be evaluated. Since, in general, a redundant manipulator can reach each of these positions with an infinite number of configurations, a set of initial configurations must be considered. This set of configurations is obtained from the discretized self-motion manifold(s) corresponding to the initial configuration. The procedure for the analysis is summarized in the following pseudocode.

${ }^{9}$ The effect of the choice of the final position on the convergence behavior, for a given initial position, can be addressed in a similar manner. 
- Generate $B_{d}$

(the union of the bounding boxes of all the self-

motion manifolds of $\mathbf{x}_{d}$, the desired task position)

-Discretize the workspace to get a set of initial positions

$\mathbf{x}_{i}(i), i=1: n_{\mathbf{x}_{i}}$

for $i=1: n_{j t s}$ (for each joint)

- Generate $S_{i}$ (the union of all the stationary

manifolds) and classify $S_{i}$ into sinks/non-sinks

end

for $i=1: n_{\mathrm{x}_{i}}$ (for each initial end-effector position)

-Generate the self-motion manifold(s) for $\mathrm{x}_{\mathbf{i}}(i)$

for $j=1: n_{\mathbf{q}_{i}}$ (for each discretized configuration on the manifold(s))

-Compute the forward trajectory $T_{j}$ to $\mathbf{x}_{d}$ for the healthy manipulator

-Compute $T_{j i n}$, the portion of $T_{j}$ inside $B_{\mathbf{x}_{d}}$

$$
\begin{aligned}
& \text { for } T_{j_{i n}} \\
& \qquad \begin{array}{r}
\text { for } i=1: n_{j t s} \text { (for each joint) } \\
\text { - Compute the intersection of the range } \\
\text { of motion of joint } i \text { over } T_{j i n} \text {, with the } \\
\text { corresponding range over sink }\left(S_{i}\right)
\end{array} \\
& \text { for } T_{j_{i n}} \cap S_{i} \\
& \qquad \text { For each discretized point on the } \\
& \text { overlap, assume a failure of joint } i \\
& \text { and check whether or not the } \\
& \quad \text { manipulator converges to } \mathrm{x}_{d}
\end{aligned}
$$

end

$\left(T_{j_{i n}} \cap S_{i}\right.$ empty for $i=1: n_{j t s} \Rightarrow$ The task is

tolerant to an arbitrary joint failure over $T_{j n}$ )

\section{end \\ end \\ end}

The data obtained from the implementation of this procedure can be interpreted in a variety of ways; however, the questions posed earlier in this section are best addressed by the following measures:

$\mathcal{R}_{100}$ : The percentage of configurations considered for $x_{i}$, for which reachability of $x_{d}$ can be guaranteed for the failure of a joint along the entire length of the nominal trajectory.
$\mathcal{R}_{\text {avg }}$ : The percentage length of the nominal trajectory for which reachability to $x_{d}$ can be guaranteed for the failure of a joint, averaged over all considered configurations.

$\mathcal{R}_{\text {sens }}$ : The difference between the maximum and minimum, over all considered configurations, of the percentage of the trajectory for which reachability to $x_{d}$ can be guaranteed for a joint failure. This measure 
reflects the sensitivity of reachability to a choice of the intial configuration corresponding to $x_{i}$.

Analogous measures for complete fault tolerance (correct convergence), over trajectory segments guaranteeing reachability, are given by $\mathcal{F}_{100}, \mathcal{F}_{\text {avg }}$, and $\mathcal{F}_{\text {sens }}$, respectively.

All measures are computed for the possibility of a failure of a specific (known) joint, as well as that of an arbitrary (unknown) joint. ${ }^{10}$

\subsection{An Illustration}

The application of the workspace analysis procedure proposed above is illustrated for the example of a 3-DOF planar manipulator with unit link lengths. The desired position considered in this example of a point-to-point motion task is $x_{d}=[1.51 .5]^{T}$. The workspace of the manipulator is sampled on an equally spaced square grid, using 2032 samples. Each of these samples is evaluated as a candidate initial position for the task. The stationary marifolds for this example are generated and partitioned into sirk and nonsink segments as discussed in Section 4.6. The bounding box $\mathcal{B}_{x_{d}}$ for the single self-motion manifold corresponding to $x_{d}$ is computed using the technique outlined in Section III. The bounds are $\left[-24.30^{\circ}\right.$ $\left.114.30^{\circ}\right],\left[-111.80^{\circ} 111.80^{\circ}\right]$, and $\left[-111.80^{\circ} 111.80^{\circ}\right]$, for joints 1, 2, and 3, respectively. In Figure 10 the workspace is shaded as a function of four of the six proposed measures: $\mathcal{R}_{100}, \mathcal{R}_{\text {avg }}, \mathcal{R}_{\text {sens }}$, and $\mathcal{F}_{100}$, considering arbitrary failures. These plots demonstrate how the failure tolerance properties of the manipulator change over the workspace. Since the measures change in a continuous manner over the workspace, these plots can be used to identify regions that guarantee a certain level of failure tolerance. It is noted that in general all regions with nonzero reachability measures $\mathcal{R}_{100}$ and $\mathcal{R}_{\text {avg }}$ exhibit high values of the corresponding comprehensive fault tolerance measures $\mathcal{F}_{100}$ and $\mathcal{F}_{\text {avg }}$ (not shown). In other words, most trajectory segments that guarantee the reachability of the task position also guarantee correct convergence. Though this is true for $x_{d}$ in this example, it may not be guaranteed for an arbitrary choice of task position. It was also seen that the comprehensive sensitivity measure $\mathcal{F}_{\text {sens }}$ (not shown) is very low over the workspace, implying that, for this example, if reachability

${ }^{10}$ When a specific joint is considered, only projections of trajectories and bounding boxes for that joint axis are relevant. is guaranteed, correct convergence has little dependence on the initial configuration of the manipulator. Figures 5 and 6 are examples of postfailure convergence behaviors that could be predicted by studying similar plots of workspace measures. While not shown here, it turns out that the initial positions of the manipulator in both Figures 5 and 6 are characterized by perfect measures of $\mathcal{R}_{100}$, i.e., $100 \%$, for the failures of joints 2 and 1 , respectively. It is the differing values of the corresponding $\mathcal{F}_{100}$ meastures of these positions that result in the different convergence behaviors. The initial position considered in Figure 5 is characterized by a high $\mathcal{F}_{100}$ measure, and so correct convergence is almost always observed for a failure. The case presented in Figure 5 is an example of such behavior. On the other hand, the initial position consicered in Figure 6 is characterized by a low $\mathcal{F}_{100}$ measure, and consequently incorrect convergence is seen for a significant number of failure configurations. An example of this problem is illustrated by the case presented in Figure 6 . Workspace plots such as those shown in Figure 10 can thus be effectively used for an off-line prediction of the postfault behavior of a manipulator.

\section{CONCLUSION}

In this work the behavior of robotic manipulators performing point-to-point motion tasks with unidentified locked-joint failures was analyzed. Conditions governing convergence issues stuch as whether the manipulator comes to rest, and if so, at what terminal position of the end-effector, were explicitly defined and the different possible convergence behaviors, illustrated with examples. A general procedure for evaluating the failure-tolerance properties of a manipulator over its workspace was also presented.11. Since no closed form expressions to guarantee correct convergence exist, brute-force evaluations to check for the effect of certain failures become necessary over some portions of the workspace. However, the procedure based on the developed analysis tools allows for an intelligent evaluation of the workspace, thereby minimizing the number of brute-force checks. Using workspace information for task placement is an effective approach to coping with failures, with or without failure identification.

\footnotetext{
${ }^{11}$ The analysis procedure can be extended, in a manner analogous to the one in ref. 27 , to design manipulators that are guaranteed to converge in the post-failure workspace.
} 


\section{REFERENCES}

1. B.S. Dhillon, Robot reliability and safety, SpringerVerlag, New York, 1991.

2. R. Colbaugh and M. Jamshidi, Robot manipulator control for hazardous waste-handling applications, J. Robot Syst, 9:(2) (1992), 215-250.

3. M.L. Visirsky, J.R. Cavallaro, and I.D. Walker, Robotic fault detection and fault tolerance: A survey, Reliab Eng Syst Safety 46 (1994), 139-158.

4. Unimation Incorporated, Unimate Puma Mark II Robot 500 Series Equipment Manual, Danbury, CT, 1985.

5. K.N. Groom, A.A. Maciejewski, and V. Balakrishnan, Real-time failure tolerant control of kinematically redundant manipulators, IEEE Trans Robot Autom 15:(6) (1999), 1109-I116.

6. J.D. English and A.A. Maciejewski, Fault tolerance for kinematically redundant manipulators: Anticipating free-swinging joint failures, IEEE Trans Robot Autom 14:(4) (1998), 566-575.

7. G. Litr, Control of robot manipulators with consideration of actuator performance degradation and failures, in 2001 Int Conf Robot Autom Seout, Korea, IEEE, 21-26 May, 2001.

8. E.C. Wu, J.C. Hwang, and J.T. Chladek, Fault-tolerant joint development for the space shuttle remote ma. nipulator system: Analysis and experiment, IEEE Trans Robot Autom 9:(5) (1993), 675-684.

9. A.K. Pradeep, P.J. Yoder, R. Mukundan, and R.J. Schilling, Crippled motion in robots, IEEE Trans Aerosp Electron Syst 24:(1) (1988), 2-13.

10. C.L. Lewis and A.A. Maciejewski, Dexterity optimization of kinematically redundant manipulators in the presence of failures, Comput Elect Eng 20:(3) (1994), 273-288.

11. C.J.J. Paredis, W.K.F. Au, and P.K. Khosla, Kinematic design of fault tolerant manipulators, Comput Electr Eng 20:(3) (1994), 211-220.

12. R.G. Roberts and A.A. Maciejewski, A local measure of fault tolerance for kinematically redundant manipulators, IEEE Trans Robot Autom 12:(4) (1996), 543-553.

13. R.G. Roberts, On the local fault tolerance of a kinematically redundant manipulator, J Robot Syst 13:(10) (1996), 649-661.

14. W.E. Dixon, I.D. Walker, and D.M. Dawson, Fault detection for wheeled mobile robots with parametric uncertainty, in 2001 Int Conf Advanced Intelligent Mechatronics. Vol. 2. IEEE/ASME, 2001, pp. 12451250.

15. M.H. Terra and R. Tinós, Fault detection and isolation in robotic manipulators via neural networks: A comparison among three architectures for residual analysis, J Robot Syst 18:(7) (2001), 357-374.

16. L. Notash, Joint sensor fault detection for fault tolerant parallel manipulators, J Robot Syst 17:(3) (2000), 149157.

17. M.L. Visinsky, J.R. Cavallaro, and I.D. Walker, A dy- namic fault tolerance framework for remote robots, IEEE Trans Robot Autom 11:(4) (1995), 477-490.

18. Y. Ting, S. Tosunoglu, and D. Tesar, A control structure for fault-tolerant operation of robotic manipulators, in 1993 Int. Conf. Robot Autom, Atlanta, GA, IEEE, 2-6 May, 1993, pp. 684-690.

19. J.E. McInroy, J.F. O'Brien, and G.W. Neat, Precise, faulttolerant pointing using a stewart platform, [EEE/ ASME Trans Mechatron 4:(1) (1999), 91-95.

20. J.E. McInroy, G.W. Neat, and J.F. O'Brien, Robotic approach to fault-tolerant, precision pointing, IEEE Robot Autom Mag 6:(4) (1999), 24-31.

21. D. Um and V. Lumelsky, Fault tolerance via analytic redundancy for a modularized sensitive skin, Int J Robot Autom 15:(4) (2000), 155-165.

22. B.M. Harpel, J.B. Dugan, I.D. Walker, and J.R. Cavallaro, Analysis of robots for hazardous environments, in Proc of the Annual Reliability and Maintainability Symposium, Philadelphia, PA, January 1997, pp. 111116.

23. S. Kimura, M. Takakashi, T. Okuyama, S. Tsuchiya, and Y. Suzuki, A fault-tolerant control algorithm having a decentralized autonomous architecture for space hypermredundant maripulators, IEEE Trans Syst Man, Cybern SMC-28:(4) (1998), 521-527.

24. C.L. Lewis and A.A. Maciejewski, Fault tolerant operation of kinematically redundant manipulators for locked joint failures, IEEE Trans Robot Autom 13:(4) (1997), 622-629.

25. C.J.J. Paredis and P.K. Khosla, Mapping tasks into fault tolerant maniputators, in 1994 Int Conf Robot Autom, San Diego, CA, IEEE, 8-13 May, 1994, Pp. 696-703.

26. F. Miyazaki and Y. Masutani, Robustness of sensory feedback control based on imperfect Jacobian, in Robotics Research: The Fifth International Symposium, H. Miura and S. Arimoto, eds. MIT, Cambridge, 1989, pp. 201-208.

27. M. Shoham and Y. Koren, Motion control algorithms for sensor-equipped robots, ASME J Dyn Syst Measure Control 109 (1987), 335-344.

28. Y. Ting, S. Tosunoglu, and B. Fernández, Control algorithms for fault-tolerant robots, in 1994 Int Conf Robot Autom, San Diego, CA, IEEE, 8-13 May, 1994, pp. 910915.

29. Y. Ting, S. Tostunoglu, R. Freeman, and D. Tesar, Saturation avoidance methods for serial robots operating tunder a failure, J Robot Syst 16:(12) (1999), 667-678.

30. E. Wu, J. Hwang, and J. Chladek, A failure tolerant joint design for the space shuttle remote manipulator system: Analysis and experiment, in First IEEE Conf Control Applications, Dayton, $\mathrm{OH}, 13-16$ September, 1992, pp. 330-335.

31. C.L. Lück and S. Lee, Self-motion topology for redundant manipulators with joint limits, in 1993 Int Conf Robot Autom, Atlanta, GA, IEEE, 2-6 May, 1993, pp. 626-631. 